\title{
Participación electoral en Chile. Una aproximación de edad, PERÍODO Y COHORTE ${ }^{*}$
}

\author{
Electoral Turnout in Chile: An Age-Period-Cohort Approach
}

\author{
MATÍAS BARGSTED \\ Pontificia Universidad Católica de Chile
}

\author{
NICOLÁS M. SOMMA
}

Pontificia Universidad Católica de Chile

\section{BENJAMÍN MUÑ̃Z ROJAS}

Centro de Estudios de Conflicto y Cohesión Social (COES), Chile

\begin{abstract}
RESUMEN
Por medio de un análisis de edad-período-cohorte, aplicado a datos de encuestas realizadas entre 1994 y 2015, buscamos comprender el declive de la participación electoral ocurrido en Chile durante la actual era democrática. Nuestro análisis confirma algunas hipótesis previas, pero también ilumina algunas nuevas tendencias. Primero, encontramos efectos de cohorte que indican que las generaciones que votaron durante el plebiscito de 1988 tienden a sufragar más que aquellas que se volvieron elegibles en democracia. Segundo, la propensión a votar de los chilenos aumenta con el envejecimiento, aunque este efecto es mucho más pronunciado entre las cohortes nacidas después de 1970. Por último, hallamos un marcado y negativo efecto período de carácter lineal, que implica un declive universal en la propensión a concurrir a las urnas de todos los grupos etarios.
\end{abstract}

Palabras clave: participación electoral, generaciones, edad, período, Chile

\begin{abstract}
Through an age-period-cohort analysis applied to survey data from 1994 to 2015, we seek to understand the decline in electoral participation that has occurred in Chile during the current democratic era. Our analysis confirms some previous hypotheses, but it also illuminates some new trends. First, we find cohort effects indicating that the generations that voted during the 1988 Plebiscite tend to vote more often than those who became eligible during the democratic era. Second, Chileans' propensity to vote increases with age, although this effect is much more pronounced among cohorts born after 1970. Finally, we find a strong and negative linear period effect, whereby the propensity to vote among all age groups has declined universally.
\end{abstract}

Keywords: electoral participation, generations, age, time period, Chile

\footnotetext{
Agradecemos al Fondo Nacional de Desarrollo Científico y Tecnológico (FondecyT)-Comisión Nacional de Investigación Científica y Tecnológica (Conicyt-Chile), por su apoyo a través del Centro de Estudios de Conflicto y Cohesión Social (COEs), al fondo ConicyT / Fondo de Financiamiento de Centros de Investigación en Áreas Prioritarias (FondAP)/15130009, el Concurso Fondecyt Iniciación 11140746 (IR Matías Bargsted) y el Concurso FondecYT Regular 1160308 (IR Nicolás Somma). El apéndice y el archivo de reproducción pueden ser descargados en https://sites.google.com/uc.cl/matiasbargsted/pagina-principal/publications
} 


\section{INTRODUCCIÓN}

Uno de los fenómenos más llamativos de la política chilena — desde la transición a la democracia en 1990 hasta la fecha- es el sostenido e ininterrumpido declive de la participación en elecciones nacionales. Mientras que en los comicios presidenciales inaugurales de 1989 acudió el 84,2\% de la población en edad de sufragar, ${ }^{1}$ en las votaciones de 2017 la cifra bajó al 46,7\% en la primera vuelta y al 49,0\% para el balotage. ${ }^{2}$ La reforma de 2012, que tuvo como propósito incentivar la participación, estableció el tránsito de una inscripción voluntaria y voto obligatorio a una de tipo automática y sufragio voluntario que fue claramente insuficiente. El descenso en la asistencia a las urnas fue uno de los más pronunciados del mundo en las últimas décadas, situando a Chile como uno de los países que registra la menor participación electoral en América Latina (Programa de las Naciones Unidas para el Desarrollo 2016, 3).

Esta tendencia es preocupante. Las democracias con baja participación electoral suelen ser más vulnerables a crisis de legitimidad pública y quienes no votan, tienden a pertenecer a grupos desaventajados de la población, facilitando que sus intereses no sean considerados por las autoridades políticas (Lijphart 1997). Corvalán y Cox (2013) muestran que, en Chile, no solo existe una brecha de participación entre ricos y pobres, sino que también entre personas mayores y jóvenes. Esto lleva a subrepresentar a los segundos a costa de los primeros y genera fuertes dudas sobre la capacidad del sistema político para canalizar las demandas de las nuevas generaciones.

En la última década, varios investigadores se volcaron a explorar por qué cayó la participación electoral en Chile. La interpretación convencional postula que el contexto político-institucional produjo generaciones de ciudadanos altamente participativas que fueron progresivamente remplazadas - a medida que avanzaron las décadas de 1990 y 2000 - por cohortes con menor propensión a votar. El resultado habría sido la caída de la participación a nivel agregado.

Esta interpretación abre varias interrogantes vinculadas a las fuentes de esta tendencia. ¿El descenso agregado en la participación electoral se explica únicamente por el remplazo generacional? $\mathrm{O}$, más bien, ¿la propensión a votar de todos los grupos etarios ha decrecido a medida que pasa el tiempo? ¿Puede que ambos procesos, reemplazo generacional y declive universal, se den simultáneamente o puede, incluso, ocurrir que a medida que pasa el tiempo, las distintas generaciones - o solo algunas de ellas- voten cada vez más, generando un efecto opuesto?

Calculado en base a datos del Servel (www.servel.cl/elecciones-presidenciales-resultados-globales/) y del INE (www.ine.cl/estadisticas/demograficas-y-vitales).

Tomado de las estadísticas de participación del Servel (https://www.servel.cl/estadisticas-de-participacion/). 
Las investigaciones previas no han podido responder cabalmente estas preguntas. Han utilizado diseños de investigación que abarcan una o muy pocas mediciones en el tiempo y no controlan simultáneamente por las tres fuentes de cambio longitudinal —edad de los entrevistados, cohorte de nacimiento y período de la encuesta-. Bajo esas condiciones, es imposible describir adecuadamente las distintas fuentes del declive agregado en la participación electoral. Aún más, la explicación tradicional, basada en generaciones, no da cuenta de la evidencia internacional que sostiene que, con el aumento de la edad, se incrementa la propensión a votar. Dado lo anterior, nuestro artículo busca comprender cómo ha evolucionado la participación electoral en Chile en base a un diseño de investigación de edad-período-cohorte (EPC), con datos provenientes de encuestas de corte transversal repetidas anualmente durante un período de 22 años (1994-2015).

Nuestras estimaciones confirman resultados previos, pero también revelan patrones desconocidos. Primero, consistente con Toro (2008) y Contreras y Navia (2013), entre otros, se observan efectos de cohorte marcadamente no lineales: aquellas personas que alcanzaron a participar del plebiscito de 1988 y, especialmente, aquellas nacidas entre 1960 y 1970, registran tasas de participación electoral más altas que quienes no alcanzaron a votar en dicha elección. Segundo, a medida en que las personas envejecen, aumenta la probabilidad de que hayan votado en una elección, aunque tal efecto es altamente heterogéneo. Son justamente los miembros de las cohortes que no alcanzaron a votar en el plebiscito de 1988 en quienes se observa el efecto etario más acentuado. Por último, observamos un fuerte efecto período de carácter lineal: aun controlando por edad y cohorte, la propensión a votar de todos los grupos etarios se ha reducido progresivamente con el paso del tiempo.

\section{DECLIVE ELECTORAL EN CHILE: INVESTIGACIONES PREVIAS}

Durante los últimos años, un número creciente de investigadores ha considerado distintos factores para comprender el declive de la participación electoral en Chile (Navia 2004; Madrid 2005; Carlin 2006; Toro 2008; Corvalán y Cox 2013; Contreras y Morales 2014). El elemento unificador de esta agenda ha sido el énfasis por la influencia de factores etarios y generacionales. Una de las primeras interpretaciones del descenso en la participación electoral se basó en una supuesta diferencia en la propensión a votar entre personas de distintas edades, donde una emergente "apatía juvenil" sería la principal explicación (Parker 2000; Navia 2004).

Hace aproximadamente una década, surgió una interpretación alternativa basada en la noción de generaciones. Existirían en Chile distintas generaciones o cohortes que, en función del momento histórico en que se produjo su socialización política, habrían quedado predispuestas a mayores o menores niveles de participación electoral, siendo este un efecto duradero en el tiempo. 
Uno de los trabajos pioneros en esta línea fue Toro (2008), quien distinguió tres generaciones en función de la edad de los participantes de la encuesta del Latin American Public Opinion Project (LAPOP) 2008: los jóvenes entre 18 y 29 años, socializados en tiempos de desafección; los adultos postplebiscito, socializados en una época de "normalización democrática" y los adultos preplebiscito, quienes experimentaron el tiempo de "epopeya" de los comicios de 1988. Estos últimos presentarían mayores niveles de participación electoral no solo por haber experimentado directamente el período épico de la transición a la democracia, sino además por haberse inscrito en forma masiva en 1988 para votar en la elección, instancia en la que quedaron cooptados por el sistema de voto obligatorio. Para las generaciones siguientes, en cambio, el plebiscito de 1988 se convertía progresivamente en un hecho histórico distante y se enfrentaban a un sistema electoral que imponía altos costos.

Consistente con esto, Contreras y Navia (2013) muestran que los niveles de inscripción electoral entre los grupos etarios que alcanzaron la adultez durante el periodo democrático fueron mucho menores que para los grupos de más edad. Mientras que en 1990, aproximadamente el 65\% de los chilenos entre 18 y 24 años se había inscrito, en 2009 —diecinueve años más tardeesta cifra se redujo a cerca de un $20 \%$. En consecuencia, a medida que pasó el tiempo, la "generación épica" habría sido progresivamente remplazada por las postplebiscito, reduciendo la proporción del electorado que vota. En forma similar, Corvalán y Cox (2013) usaron encuestas de LAPOP en tres olas —2006, 2008 y 2010 - y distinguieron entre las cohortes de "alta votación" que serían remplazadas por los de "baja votación". Concluyeron que la disminución de la participación electoral en Chile "se debe, sin lugar a dudas, al remplazo generacional" (Corvalán y Cox 2013: 53).

Aunque la tesis generacional ha encontrado amplio eco entre las investigaciones sobre el declive electoral chileno, el mecanismo exacto que explica tales diferencias no ha sido enteramente aclarado. Algunos autores han enfatizado las diferencias culturales y niveles de politización que se habrían producido entre las cohortes pre y postplebiscito. Por ejemplo, Carlin (2006) dividió a los entrevistados de una encuesta del Centro de Estudios Públicos (CEP) en cuatro "generaciones políticas" según su edad. Encontró que las dos generaciones que se volvieron elegibles pata votar en democracia (que tenían entre 18 y 23, y 24 y 31 años en 1999, respectivamente) votaban en menor medida que la generación que fue elegible para votar en el Plebiscito de 1988 (de 32 a 43 años) o en 1973 (44 años o más en 1999), y atribuyó la diferencia a un "cambio generacional" en la cultura política chilena que alejaba a las generaciones recientes de actividades colectivas. Toro (2008) también pareciera favorecer esta perspectiva al demostrar que, entre los adultos preplebiscito y los jóvenes socializados en democracia, hay marcadas diferencias en sus niveles de confianza institucional y orientaciones ideológicas. Por otro lado, Contreras y Navia (2013) adoptan una posición más institucionalista al argumentar que los contrastes generacionales se explican 
únicamente por la menor tasa de inscripción de las cohortes socializadas en democracia.

En oposición con la prominencia de la tesis del remplazo generacional, en la discusión nacional, la tesis del "envejecimiento" ha tenido mucho menos espacio, según la cual, la propensión a votar aumenta con el incremento de la edad de las personas. Dicha hipótesis, de gran importancia en la literatura internacional, también implicaría observar diferencias en los niveles de participación entre las cohortes pre y postplebiscito, en la medida en que los primeros son más jóvenes que los segundos. A continuación, revisamos esta literatura.

\section{EFECTO ETARIO EN LA LITERATURA INTERNACIONAL}

La literatura comparada ha enfatizado el efecto positivo de la edad sobre la participación electoral, distinguiéndolo explícitamente de las diferencias generacionales (Blais 2000; Dassonneville 2017) ${ }^{3}$. En un meta-análisis reciente, Smets y van Ham (2013) concluyen que en un 75\% de los estudios analizados sobre participación electoral, la edad tiene un efecto sustantivo sobre la propensión a votar. La evidencia también es robusta en describir la relación entre edad y participación como una línea curva: los jóvenes son los que tienen mayor probabilidad de abstenerse $\mathrm{y}$, a medida que envejecen, aumenta su propensión a participar, para luego volver a decrecer gradualmente en edades más avanzadas. Este patrón se presenta en democracias estables y emergentes (Plutzer 2018), incluyendo América Latina (Carlin y Love 2015).

La investigación se ha centrado en dos enfoques para explicar este vínculo. Primero, la teoría del ciclo de vida político enfatiza que las distintas etapas de la adultez implican variadas demandas sobre los individuos, alterando los motivos, oportunidades y recursos necesarios para participar (Strate et al. 1989). El principal mecanismo causal de este enfoque se centra en experiencias vitales vinculadas a la transición a la adultez (Plutzer 2018, 72). Los jóvenes son menos propensos a participar ya que se ven presionados por distintos "problemas de arranque" (Goerres 2007) como los estudios superiores, la búsqueda de pareja, el ingreso al mercado laboral y el abandono del hogar paterno. De este modo, la edad sería un proxy de estos "indicadores de maduración" (Highton y Wolfinger 2001). Posteriormente, los individuos adquirirían más recursos y motivos para participar. Otro mecanismo se centra en las "experiencias políticas", enfatizando la familiaridad con la política y las redes sociales. Al avanzar a la adultez, los individuos fijan su residencia, forman familia y establecen mayores vínculos comunitarios. Lo anterior aumenta el interés en la política y el compromiso cívico (Flanagan et al. 2012; Smets 2016), incrementando la participación. Luego, con la vejez llega la desvinculación del mercado laboral, el deterioro de

3 Los efectos cohorte difieren según país. Grasso (2014) detecta, al interior de Europa, distintos patrones de descenso. En Estados Unidos, las diferencias generacionales no son lineales. 
la salud y la pérdida de familiares y amigos involucrados políticamente, lo cual repercute en un menor arraigo político y propensión a votar.

Un segundo enfoque explicativo sostiene que el voto opera como un hábito repetitivo $^{4}$ (Milbrath 1965; Verba y Nie 1972; Dinas 2012), de modo que sufragar en una elección aumentaría la probabilidad de hacerlo en elecciones sucesivas, tras controlar por otros factores individuales (Czesnik et al. 2013, 99). Con los años -y la sucesiva exposición a elecciones-, los individuos acumulan información sobre el sistema político y, gradualmente, van aclarando sus intereses y preferencias, reduciendo los costos de participar (Denny y Doyle 2009; Plutzer 2002, 2018). Esto se ve complementado por un proceso psicológico en el que acudir a las urnas refuerza las actitudes cívicas de los ciudadanos e incrementa su autoimagen como "votantes", aumentando su sensación de eficacia política y comodidad con el acto de votar (Green y Shachar 2000).

Es probable que estos distintos procesos operen simultáneamente. Zeglovits y Aichholzer (2014) detectan que es más probable que vote por primera vez un joven de 16 años que uno de 20, ya que el primero aún vive con sus padres e interactúa con otros miembros de su comunidad que sí son votantes. En cambio el de 20 años, tras abandonar el hogar y comenzar los estudios superiores, se encuentra inserto en una red mayoritariamente de no votantes. De todos modos, una vez que ambos jóvenes hayan votado por primera vez, es más probable vuelvan a hacerlo en el futuro. No es nuestro objetivo distinguir analíticamente entre ciclo vital y hábito, pero destacamos que ambos sustentan un efecto positivo de la edad en las chances de votar.

\section{LA NECESIDAD DE UN NUEVO ABORDAJE}

Sintetizando la discusión anterior ¿cómo podemos estudiar los múltiples procesos de estabilidad y cambio que subyacen al patrón agregado de declive electoral en Chile? ¿Cómo reconocer si el descenso se debe únicamente al remplazo generacional o si este proceso está acompañado por una tendencia universal de todos los grupos etarios a votar con menor frecuencia? ¿Es factible que haya, además, una tendencia "subterránea", de sentido opuesto, por la cual las generaciones votan más a medida que pasa el tiempo?

Para abordar estas preguntas, se requiere clarificar las posibles fuentes de cambio social. Siguiendo a Firebaugh (1997), definimos "cambio social" como aquel que se realiza a nivel agregado de algún atributo o comportamiento colectivo de un grupo de personas, como la tasa de participación electoral en Chile. Desde una perspectiva EPC, hay dos fuentes de cambio social: las personas mismas pueden cambiar o cambia la composición de la población.

Se debate si es el concepto más adecuado, dada la escasa frecuencia con que los ciudadanos votan (una vez cada o dos tres años). De ahí que se han propuesto otros conceptos como costumbre (Green y Shachar 2000), inercia (Plutzer 2002) y persistencia (Denny y Doyle 2009). 
El primer tipo de cambio puede, a su vez, ser dividido en "efecto etario" $\mathrm{y}$ "efecto período". El primero refiere a las variaciones asociadas al transcurrir del ciclo de vida de las personas. En nuestro caso, describe los cambios en los niveles de participación electoral que ocurren con el incremento de la edad. Por su parte, los segundos reflejan variaciones en el tiempo (años en nuestro análisis) en el nivel de participación electoral que ocurren entre todos los grupos etarios de forma simultánea. Estos efectos pueden deberse al impacto de acontecimientos macrosociales específicos, o bien, pueden capturar tendencias sistemáticas a las cuales están expuestos todos los miembros de la sociedad (Yang y Land 2013).

La segunda fuente de cambio social — cambio en la composición de una sociedad - es capturada por medio del efecto cohorte: la composición cambia porque las cohortes más antiguas mueren y dan paso a las más jóvenes, produciéndose un remplazo generacional. En nuestro caso, estos efectos reflejan variaciones en los niveles de participación electoral entre grupos de personas que se definen por el hecho de haber experimentado los mismos acontecimientos históricos y sociales a edades similares, especialmente durante su juventud y adultez temprana. Los eventos ocurridos durante dichos "años impresionables" (Krosnick y Alwin 1989), tienen efectos duraderos sobre las actitudes y conductas de los individuos de una misma generación. Para el caso chileno, es particularmente importante diferenciar el efecto cohorte del efecto período: el descenso observado puede ser el producto simultáneo de la entrada de nuevas cohortes que votan menos y de un proceso de creciente desafección generalizado, en el que una proporción cada vez mayor de miembros de todas las cohortes dejan de votar progresivamente.

Las investigaciones existentes, lamentablemente, no distinguen entre estas fuentes de cambio social. Si dividimos a los encuestados de una encuesta tomada en un solo momento en el tiempo y los etiquetamos como "pre" y "postplebiscito" - y afirmamos que los primeros votan en mayor proporción por haber estado expuestos a la épica de los 1980—, no estamos considerando la posibilidad de que voten más simplemente porque son más viejos. En este caso, no es posible distinguir el efecto etario del efecto cohorte, ya que ambas variables son perfectamente colineales entre sí (Mason et al. 1973). Evidentemente, tampoco es posible desentrañar un efecto período si es que se ha observado la participación electoral en un solo punto en el tiempo.

Los pocos estudios nacionales que consideran encuestas en más de un solo punto en el tiempo (Madrid 2005; Corvalán y Cox 2013) ciertamente son un avance. Sin embargo, cubren intervalos temporales acotados o no incluyen especificaciones estadísticas que controlen simultáneamente por edad, período y cohorte. En contraste, aquí empleamos un diseño sustentado en datos anuales provenientes de encuestas transversales repetidas, que cubren un período de 22 años —desde 1994 hasta 2015-, junto con modelos de regresión jerárquicos que incorporan simultáneamente las tres fuentes de cambio social. Al emplear datos de encuestas repetidas, en las que se observan personas de una misma generación a distintas edades o en diferentes períodos (o viceversa), es posible 
romper la dependencia lineal que hay entre las tres fuentes de cambio social y, por ende, estimar el efecto separado de cada una. Hasta donde llega nuestro conocimiento, este es el primer estudio del comportamiento electoral en Chile y América Latina con estas características.

\section{HIPÓTESIS}

Dados los antecedentes teóricos y empíricos mencionados, proponemos cuatro hipótesis para evaluar empíricamente.

H1: Siguiendo la literatura nacional, sostenemos que las personas nacidas en 1970 o antes y que, por tanto, alcanzaron a inscribirse y votar en el plebiscito de 1988, tendrán una mayor propensión promedio a votar que aquellas nacidas después de ese año.

H1A: Siguiendo la noción de los años impresionables, los miembros de cohortes nacidas entre los años 1961 y 1970 - y que por ende participaron del plebiscito de 1988 durante sus años formativos - tendrían la mayor propensión promedio a votar durante el periodo bajo observación.

H2: Controlando por la cohorte de nacimiento, la participación electoral seguirá un patrón etario cuadrático: aumentará hasta cierto punto, a medida que las personas envejezcan, luego del cual comenzará a decrecer.

H2A: El efecto etario estará influido por las reglas electorales que operaron para distintas cohortes. Las cohortes nacidas en 1970 o antes se inscribieron masivamente para el plebiscito de 1988 y, dada la normativa imperante hasta la reforma de 2012, fueron obligadas a seguir votando. Por tanto, deberíamos observar débiles efectos de la edad para estas cohortes. Por el contrario, el efecto de la edad se magnificará en las cohortes nacidas en 1971 o después.

\section{MEDICIÓN Y DATOS EMPÍRICOS}

Emplearemos 37 encuestas de corte transversal realizadas por el Centro de Estudios Públicos (CEP) entre 1994 y 2015. Ellas utilizan un diseño muestral probabilístico multietápico por conglomerados y son representativas de la población chilena adulta (mayores de 18 años). ${ }^{5}$

Nuestra variable dependiente indica si el entrevistado mencionó haber votado o no en la última elección presidencial y / o legislativa realizada en el país durante el período de aplicación de la encuesta. Esta variable tiene dos problemas. 
Por un lado, la pregunta sobre participación electoral no ha sido medida de forma idéntica en todas las encuestas. El formato más común (aplicado en 21 de 25 encuestas) es: " ¿Votó usted en la pasada elección presidencial y parlamentaria del [mes/año]?". En otras tres ocasiones se aplicaron preguntas similares como "¿Concurrió usted a votar en las últimas elecciones presidenciales del [día/mes/año]?". Y en otro momento (2014) se consultó del siguiente modo: "Ahora me gustaría saber cuál fue su decisión. En la pasada elección presidencial, ¿usted se abstuvo, es decir, no votó en la elección presidencial?, ¿o usted si votó en la elección presidencial?". Creemos que no hay nada evidente en la formulación de las preguntas que sugiera que puedan registrar tasas de participación sistemáticamente diferentes entre ellas y, por ende, las diferencias en el formato de medición no inhiben el uso longitudinal de los datos. ${ }^{6}$ Debe destacarse que todas estas preguntas tienen una naturaleza retrospectiva, las cuales presentan menores sesgos de deseabilidad social en comparación a preguntas de intención de participar en la siguiente elección (Smets y van Ham 2013).

El segundo problema supone mayores desafíos. El CEP no incluyó preguntas de participación electoral en todas sus encuestas. Dicha ausencia de información plantea una dificultad de datos perdidos, interrumpiendo la periodicidad de la serie temporal. Sin embargo, el CEP sí incorporó, en la mayoría de sus encuestas realizadas durante el período 1994-2012, una pregunta acerca de si el encuestado estaba inscrito para votar. Dado el sistema institucional vigente en dichos años, la inscripción es un sólido predictor del comportamiento electoral (Contreras y Navia 2013). En términos empíricos, la correlación policórica entre estar inscrito y haber votado es 0,95 .

Utilizamos la variable de inscripción electoral para recuperar, vía imputación múltiple (Van Buuren 2012), el registro de participación electoral de aquellos entrevistados de las encuestas CEP que excluyeron esta pregunta del cuestionario. Por medio de la imputación, recuperamos encuestas de los años 1994, 1995, 1996, 2004 y 2009, manteniendo la periodicidad anual de la serie. El modelo de imputación utilizado incluye como predictores, además de la inscripción electoral, nivel educacional y cohorte de nacimiento de los entrevistados. Este modelo se ajusta muy bien a los datos: predice correctamente el 95\% de las observaciones y obtiene un pseudo $R^{2=0,66}$. Aún más, la variable con mayor poder predictivo (inscripción electoral) no es utilizada en los modelos analíticos, resolviendo una de las críticas más frecuentes a la imputación múltiple (Von Hippel 2007). ${ }^{7}$

Las variables independientes clave de nuestros modelos analíticos de participación electoral son: edad del encuestado, año de la encuesta (período)

6 Durante 2014 se aplicaron dos encuestas CEP. Una empleó la pregunta más comúnmente usada y la otra esta versión alternativa. A pesar del cambio de redacción, ambas registraron un nivel de participación electoral similar y estadísticamente equivalente $(67 \%$ y $64 \%$ con datos ponderados: $t=1,62 ; p=0,13)$.

Todos los detalles acerca del algoritmo empleado, así como del modelo de imputación, se encuentran disponibles en el Apéndice en línea. 
y cohorte de nacimiento. La edad es medida como la edad declarada del encuestado dividida por diez, con rangos de 1,8 a 8,5. ${ }^{8}$ En cuanto al período, se incluye una variable que contabiliza el año en que fue aplicada la encuesta. Respecto a la cohorte de nacimiento, muchos estudios suelen distinguir generaciones históricas que agrupan a un conjunto amplio de personas expuestas a edades similares a determinados eventos y procesos históricos (Manheim 1952). El problema de esta estrategia es que requiere de supuestos teóricos acerca de la definición de los períodos críticos de la socialización. Como alternativa, y siguiendo los estándares en demografía (Yang y Land 2013), hemos construido 16 cohortes de nacimiento de cinco años que van desde aquellos nacidos entre 1921 y 1925 hasta aquellos nacidos entre 1991 y 1995. Dada su naturaleza más desagregada, estos indicadores de cohortes pueden capturar cualquier fenómeno de agrupamiento o dinámica generacional más amplia. De todos modos, definimos puntos de corte para las cohortes muy similares a los empleados por Toro (2008), quien distingue entre los nacidos hasta 1970 (generación preplebiscito), entre 1971 y 1978 (adultos postplebiscito) y aquellos nacidos en 1979 o después. En nuestro caso, y para no romper el esquema de cohortes de cinco años, distinguimos nueve cohortes de cinco años nacidas en 1970 o antes, dos cohortes nacidas entre 1971 y 1980 y tres cortes nacidas entre 1981 y 1995.

Adicionalmente, controlamos por educación y sexo de los entrevistados, ya que la literatura sugiere que ambas variables influyen sobre la participación electoral (Smets y van Ham 2013). Sexo es una variable dicotómica ( $0=$ hombre y $1=$ mujer) y educación es una variable ordinal de ocho tramos que varían desde 0 a 3 años de escolaridad hasta 18 o más años de escolaridad.

\section{VII.ESTRATEGIA ANALÍTICA Y MODELO ESTADÍSTICO}

La dependencia lineal exacta entre edad, período y cohorte plantea un severo problema de identificación. Recientemente, Yang y Land (2013, cap. 7) propusieron estimar estos efectos a través de modelos jerárquicos de clasificación cruzada aplicados a datos de encuestas repetidas de corte transversal. Esta influyente aproximación captura simultáneamente los impactos de cohorte y período como efectos aleatorios (son variables de agrupamiento) y el de la edad como un efecto fijo (variable individual).

El modelo jerárquico propuesto tiene múltiples ventajas. Permite introducir fácilmente variables de control en los modelos o transformaciones no lineales de las variables, así como evaluar la influencia de variables sustantivas que predicen cambio en los efectos período y cohorte. Sin embargo, esta estrategia

Esta codificación facilita que el proceso de estimación converja en forma más rápida. Se han excluido del análisis todos aquellos entrevistados que, cuando fueron entrevistados, no tenían edad suficiente para votar en la elección sobre la que se les consultó. 
de modelamiento tiene restricciones: es necesario asumir que el efecto aleatorio de período y cohorte no están correlacionados con los predictores de nivel individual (incluyendo edad). Salvo el caso en que el efecto cohorte sea marcadamente no lineal, los efectos aleatorios de cohorte y la edad de los entrevistados estarán correlacionados, redundando en un potencial sesgo de los coeficientes estimados (Raudenbush y Bryk 2002). En forma similar, en el contexto de una población que envejece progresivamente, tal como es el caso en Chile, efectos de período sistemáticamente decrecientes podrían estar correlacionados con la edad, constituyéndose en una segunda fuente de sesgo.

Dado este potencial problema, hemos estimado tres modelos EPC con distintas especificaciones respecto al propuesto por Yang y Land (2013). Ellos nos permiten evaluar la robustez de nuestros resultados. La estructura básica de estos se puede representar matemáticamente del siguiente modo:

$$
\begin{array}{r}
\ln \frac{\operatorname{Pr}\left(\text { Voto }_{i j k}=1\right)}{1-\operatorname{Pr}\left(\text { Voto }_{i j k}=1\right)}=\beta_{0}+\beta_{1} \text { Edad }_{i j k}+ \\
\beta_{2} \text { Edad }_{i j k}^{2}+\gamma_{j}+\beta_{3} A \tilde{\mathrm{n}} o_{k}+\mu_{k}+ \\
\beta_{4} \text { Educacion }_{i j k}+\beta_{5} \text { Mujer }_{i j k}
\end{array}
$$

donde $\operatorname{Pr}\left(\operatorname{Voto}_{i j k}=1\right)$ es la probabilidad de votar del individuo $i$, perteneciente a la cohorte $j$, y entrevistado en el período $k$. En la ecuación (1), los coeficientes $\beta$ son estimados, siguiendo la terminología de modelamiento multinivel (Raudenbush y Bryk 2002) como "efectos fijos". Esto es, efectos que son constantes para todos los individuos incluidos en la muestra. Los parámetros $\gamma_{j}$ capturan los niveles de participación electoral asociados a cada una de las $j$ cohortes de nacimiento y son estimados en el Modelo 1 como efectos fijos, donde la cohorte más antigua sirve como categoría de referencia. Luego, descomponemos los efectos período en un término fijo asociado a un predictor temporal lineal (año de la encuesta centrada en 1993) y uno aleatorio residual que captura las desviaciones anuales respecto al predictor lineal de tiempo. Esta especificación permite capturar directamente la tendencia lineal decreciente en las tasas de participación electoral observable en los datos de las encuestas CEP, pero también — vía los efectos aleatorios- las variaciones sistemáticas o fortuitas en los niveles de participación que declaran los encuestados cada año. Después de contabilizar por el patrón de descenso, es muy improbable que los efectos aleatorios residuales estén asociados a la edad de los entrevistados y, en consecuencia, evitamos cualquier riesgo de estimación sesgada.

Luego, en el modelo 2, los parámetros $\gamma_{j}$ son estimados como efectos aleatorios, donde se asume que estos se distribuyen de acuerdo al supuesto paramétrico común: $\gamma_{j} \sim N\left(0, \sigma_{\gamma}^{2}\right)$. Dicha especificación se asemeja más al modelo propuesto por Yang y Land (2013, cap. 7), donde los encuestados no solo se encuentran anidados en períodos de tiempo, sino también en cohortes. Aunque esta formulación es vulnerable al supuesto de independencia entre la edad y los efectos cohortes, tiene la ventaja de ser estadísticamente más eficiente que un modelo con solo efectos fijos (Raudenbush y Bryk 2002; Yang 
y Land 2013). El tercer modelo flexibiliza la especificación anterior al permitir que los coeficientes de las variables edad $\left(\beta_{1}\right)$ y edad al cuadrado $\left(\beta_{2}\right)$ varíen aleatoriamente entre las cohortes. Estrictamente, se reemplazan los efectos fijos de edad por $\beta_{1 j}=\beta_{1}+\delta_{1 j}$ y de edad al cuadrado por $\beta_{2 j}=\beta_{2}+\delta_{2 j}$, donde $\beta_{1}$ y $\beta_{2}$ corresponden al efecto promedio de cada variable. Los términos aleatorios $\delta_{j}$ capturan las desviaciones intercohortes del efecto promedio. ${ }^{9}$ Esta especificación nos permite examinar si el efecto de la edad es heterogéneo entre distintas cohortes.

Todos los modelos son estimados de acuerdo a diez bases de datos completadas vía imputación múltiple. Mientras los coeficientes de regresión son calculados a partir del promedio de los coeficientes estimados para cada una de las bases de datos mencionadas, los errores estándar son corregidos en forma proporcional a la incertidumbre asociada a la imputación usando las fórmulas propuestas por Rubin (2004).

\section{RESULTADOS}

Previo a la discusión de los modelos estadísticos de edad-período-cohorte, es pertinente dilucidar los principales patrones descriptivos. La Figura 1 muestra la relación entre participación electoral retrospectiva y año (período) de la encuesta (panel izquierdo), cohorte de nacimiento (panel central) y edad en tramos (panel derecho). Lo primero a destacar es el continuo descenso en la participación durante el lapso temporal estudiado (panel izquierdo). También es evidente que hay un sobrereporte de la participación electoral en comparación con los resultados oficiales, pero dicha diferencia es relativamente constante. Aún más, las pendientes de las rectas asociadas a la participación electoral en base a las respuestas en las encuestas CEP y los registros oficiales (tomados de Contreras y Navia 2013) son prácticamente idénticas. Producto de lo anterior, si bien puede existir sesgo de autoreporte, este es presumiblemente constante entre encuestas. 
Figura 1. Participación electoral en elecciones presidenciales y/o legislativas según año, cohorte y edad
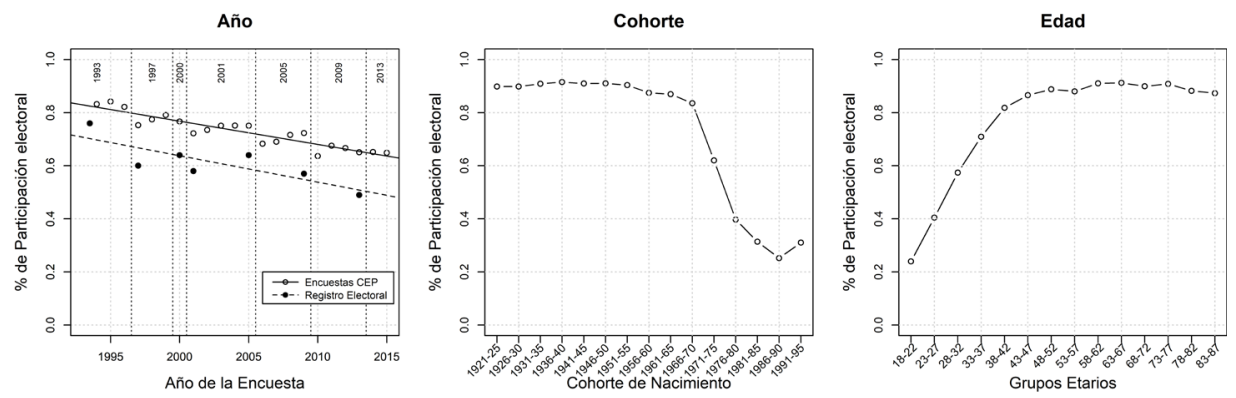

Fuente: elaboración propia en base a encuestas CEP (1994-2015)

Al analizar las diferencias entre cohortes (panel central), se aprecia que los niveles de participación para los nacidos antes de 1970 son altos y homogéneos entre sí, existiendo un patrón descendente en generaciones posteriores, con una leve recuperación para los nacidos entre 1991 y 1995. Por último, al examinar la edad del entrevistado (panel derecho), se constata un patrón opuesto al observado con las cohortes: al envejecer aumenta la propensión a votar, hasta la etapa entre 43 y 47 años, donde se estabiliza en niveles superiores al 80\%. Estos patrones opuestos evidencian la colinealidad entre edad y cohorte, resaltando la importancia de incorporar simultáneamente ambas variables en el análisis para subsanar sesgos por variable omitida.

La Figura 2 avanza en dicha dirección y muestra la evolución de los niveles de participación electoral desagregada según cohorte. En el eje horizontal se presenta el año de la encuesta, por lo que puede explorarse la evolución temporal de cada generación. Las rectas punteadas corresponden al año de la elección consultada en las encuestas. En cada subgráfico, incluimos una curva de ajuste local que captura la tendencia central de cada cohorte, con el objetivo de facilitar la visualización de patrones. Se aprecia que las cohortes nacidas en 1970 o antes muestran altos niveles de participación. Para cada una de estas diez cohortes, el promedio de participación electoral supera el $85 \%$ y se mantiene de forma estable, aunque con una leve disminución desde 2009 en adelante. Por otro lado, las cohortes nacidas a partir de 1971, que incluyen a personas que se volvieron elegibles para votar en elecciones democráticas, muestran niveles más bajos de participación electoral. Sin embargo, dichas cohortes presentan una tendencia creciente en los niveles de participación con el paso de los años. Más aún, la pendiente pareciera volverse más pronunciada al avanzar entre cohortes, indicando heterogeneidad en este efecto. Aunque estas tendencias sean reveladoras, ellas mezclan posibles efectos período con efectos etarios. Para diferenciar las tres fuentes de cambio social, recurrimos a los modelos de regresión multivariados. 

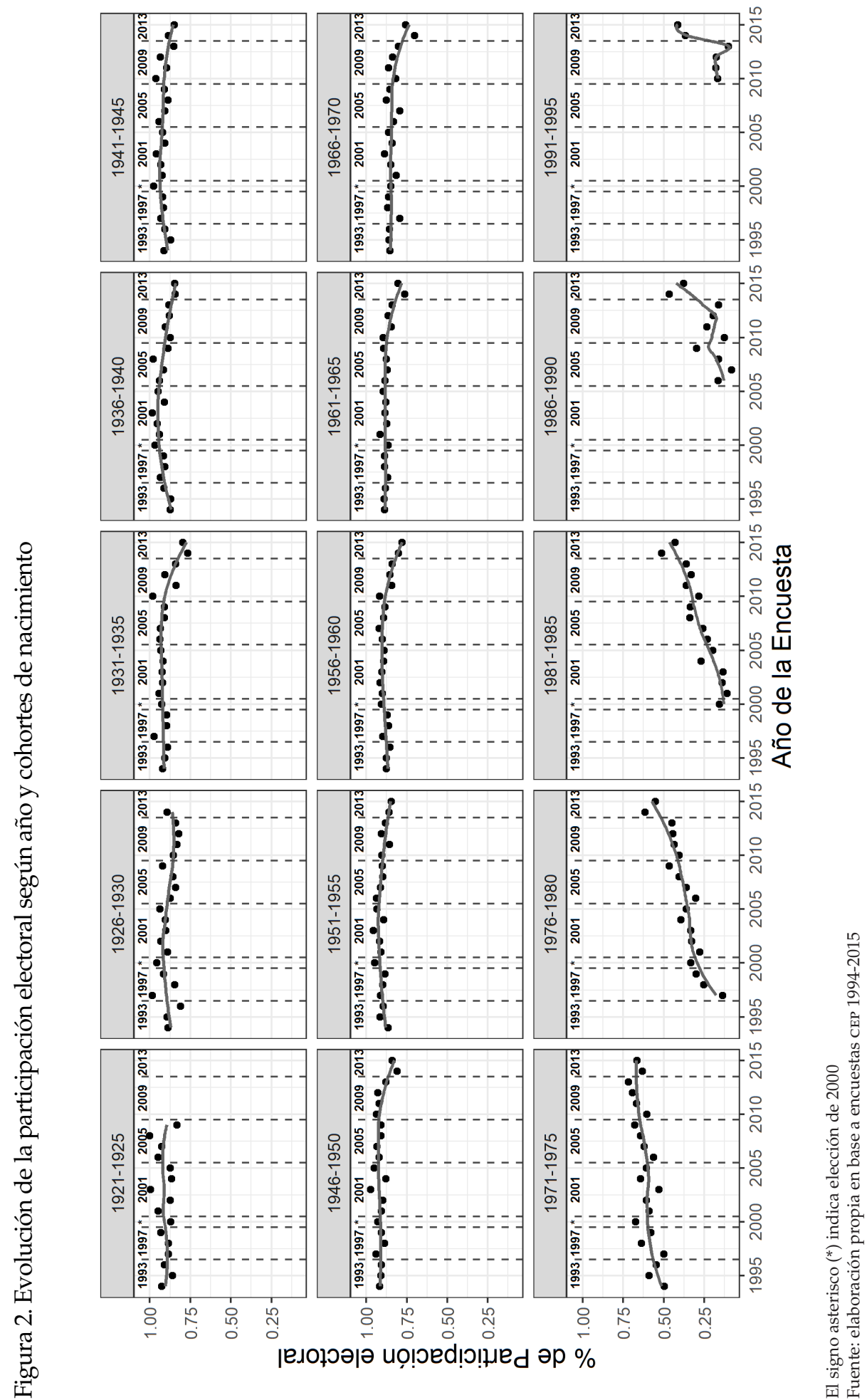
La Tabla 1 presenta los resultados de los modelos logísticos jerárquicos asociados al análisis EPC de participación electoral en Chile. En el modelo 1, la cohorte de nacimiento se estima como un efecto fijo tomando como categoría de referencia a los nacidos entre 1921-1925. Los coeficientes de las cohortes muestran una tendencia lineal creciente hasta los nacidos entre 1966 y 1970, correspondiendo a los últimos encuestados que pudieron votar en el plebiscito de 1988. Comparado con el grupo de referencia, los coeficientes de todas las cohortes posteriores a los nacidos entre 1936 y 1940 son significativos $(p<0,05)$. En cambio, para las cohortes nacidas a partir de 1971, los coeficientes estimados son más pequeños y no son estadísticamente significativos.

Tabla 1. Modelos logit jerárquicos de EPC prediciendo participación electoral

\begin{tabular}{|c|c|c|c|}
\hline & Modelo 1 & Modelo 2 & Modelo 3 \\
\hline Intercepto & $1,30(0,28)^{* * * *}$ & $1,88(0,13)^{* * *}$ & $1,73(0,13)^{* * * *}$ \\
\hline Edad & $0,70(0,09)^{* * *}$ & $0,67(0,04)^{* * *}$ & $0,62(0,08)^{* * * *}$ \\
\hline Edad $^{2}$ & $-0,12(0,01)^{* * *}$ & $-0,12(0,01)^{* * *}$ & $-0,15(0,03)^{*+*+}$ \\
\hline Educación & $0,13(0,01)^{* * * *}$ & $0,13(0,01)^{* * *}$ & $0,12(0,01)^{* * * *}$ \\
\hline Mujer & $0,06(0,03)^{*}$ & $0,05(0,03)^{*}$ & $0,05(0,03)$ \\
\hline Año & $-0,07(0,01)^{* * * *}$ & $-0,07(0,01)^{* * * *}$ & $-0,05(0,01)^{* * *+}$ \\
\hline Cohorte 1926-1930 & $0,15(0,16)$ & & \\
\hline Cohorte 1931-1935 & $0,43(0,18)^{*}$ & & \\
\hline Cohorte 1936-1940 & $0,56(0,20)^{* * *}$ & & \\
\hline Cohorte 1941-1945 & $0,66(0,24)^{* *}$ & & \\
\hline Cohorte 1946-1950 & $0,75(0,28)^{* * *}$ & & \\
\hline Cohorte 1951-1955 & $0,85(0,32)^{* *}$ & & \\
\hline Cohorte 1956-1960 & $0,85(0,36)^{*}$ & & \\
\hline Cohorte 1961-1965 & $1,14(0,40)^{* *}$ & & \\
\hline Cohorte 1966-1970 & $1,25(0,43)^{* * *}$ & & \\
\hline Cohorte 1971-1975 & $0,59(0,47)$ & & \\
\hline Cohorte 1976-1980 & $0,07(0,51)$ & & \\
\hline Cohorte 1981-1985 & $0,11(0,55)$ & & \\
\hline Cohorte 1986-1990 & $0,29(0,59)$ & & \\
\hline Cohorte 1991-1995 & $1,00(0,63)$ & & \\
\hline Período $\sigma_{\mu}^{2}$ & 0,05 & 0,05 & 0,07 \\
\hline Cohorte $\sigma_{\gamma}^{2}$ & & 0,37 & 0,28 \\
\hline Cohorte $\sigma_{E d a d}^{2}$ & & & 0,26 \\
\hline Cohorte $\sigma_{E d a d^{2}}^{2}$ & & & 0,07 \\
\hline Devianza & $44.299,68$ & $44.372,60$ & $44.185,96$ \\
\hline AIC & $44.341,68$ & $44.388,60$ & $44.211,96$ \\
\hline $\mathrm{N}$ cohortes & & 15 & 15 \\
\hline $\mathrm{N}$ obs. & 51.087 & 51.087 & 51.087 \\
\hline N períodos & 22 & 22 & 22 \\
\hline
\end{tabular}

${ }^{* * *} \mathrm{p}<0,001 ;{ }^{* *} \mathrm{p}<0,01 ;{ }^{*} \mathrm{p}<0,05$

Fuente: elaboración propia en base a encuestas CEP 1994-2015 
Estos patrones pueden verse con mayor claridad en el panel izquierdo de la Figura 3. Esta representa, en base al modelo 1, cómo varía la probabilidad promedio de participación electoral según cohorte, mientras las demás variables se fijan en sus valores observados. ${ }^{10}$ Hay un crecimiento sostenido en la probabilidad de votar entre la cohorte más antigua (1921-1925) y la última que alcanzó a votar en el plebiscito de 1988 (1966-1970). La diferencia en las probabilidades promedio entre ambas alcanza los 0,19 puntos. Entre las cohortes siguientes se aprecia un notorio descenso en las probabilidades de participación, particularmente entre aquellas que alcanzan la mayoría de edad durante la última década del siglo XX (1971-1975 y 1976-1980). Finalmente, hay un leve repunte en la cohorte más joven (1991-1995) que coincide gruesamente con quienes vivieron sus años impresionables en las movilizaciones estudiantiles de 2006 y 2011. No obstante, y tal como se aprecia en la Tabla 1, el coeficiente de esta cohorte no alcanza significancia estadística $(p=0,11)$. Más aun, esta cohorte solo ha sido observada durante seis años en nuestra base de datos, por lo que este ascenso en la tasa de participación electoral debiese considerarse con cautela.

Los resultados de las cohortes corroboran, al menos parcialmente, nuestra primera hipótesis. Con la excepción de la cohorte más joven, se confirma que las generaciones que votaron durante el plebiscito de 1989 tienen niveles de participación electoral más altos que aquellas que se volvieron elegibles en democracia. Pero las estimaciones también indican que estas últimas no se comportan en forma homogénea, existiendo patrones de crecimiento lineal y posterior caída de las probabilidades de votar. Luego, en concordancia con la hipótesis 1a, aquellos que vivieron la campaña del plebiscito de 1988 durante sus años impresionables (nacidos entre los años 1960-1965 y 1966-1970), registran la tasa de participación promedio más alta durante el período estudiado.

10 Las probabilidades predichas de las Figuras 3 y 4 han sido calculadas en base al método de los valores observados (Hanmer y Ozan Kalkan 2013). Los cálculos de probabilidades predichas condicionales (donde las demás variables independientes se fijan en su valor promedio o modal) llevan a estimaciones muy similares. 
Figura 3. Probabilidades predichas promedio de participación electoral según cohorte y período
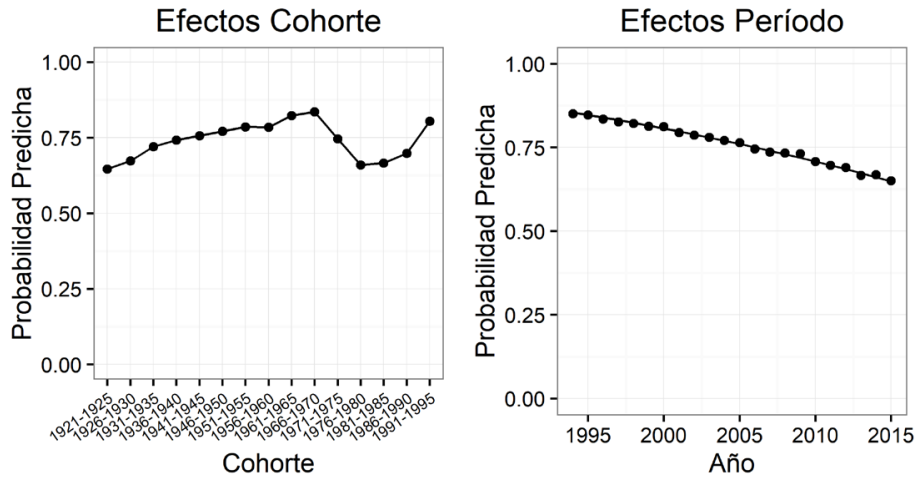

Fuente: elaboración propia en base a encuestas CEP (1994-2015)

Pasando al efecto período, el modelo 1 también indica que el predictor lineal de tiempo (variable año) tiene un impacto negativo y estadísticamente muy significativo $(p<0,001)$, lo que implica que la propensión a votar de todos los grupos etarios disminuyó con el paso del tiempo. En contraste, la variación de los efectos aleatorios asociados al año de la encuesta es muy acotada (), por lo que, tras controlar por el referido predictor lineal, no hay grandes variaciones anuales en la participación. Lo anterior se sintetiza en la Figura 3 (panel derecho), que presenta la probabilidad promedio de votar según el año: la línea representa los resultados basados únicamente en el predictor lineal y los puntos incorporan las variaciones capturadas por los efectos aleatorios. Debe destacarse que el descenso en el nivel de participación es bastante agudo, pasando de 0,85 en 1994 hasta 0,65 veintiún años más tarde. Esto implica que el descenso agregado no solo se explica por la menor propensión a votar de las cohortes postplebiscito, sino también por un comportamiento similar generalizado de todos los grupos etarios de la sociedad chilena. También es interesante notar que los efectos aleatorios asociados a la elección de 2013, la primera votación presidencial y legislativa con el sistema de voto voluntario, no se desvían particularmente de la predicción lineal. Esto implica que el cambio a voto voluntario y registro automático no generó discontinuidades en el patrón de declive, al menos cuando se lo considera a la luz de la razón entre número de votantes y personas elegibles para votar. ${ }^{11}$

Antes de examinar el efecto etario en la propensión a votar, es relevante una precisión metodológica. El modelo 2 es similar al 1, diferenciándose únicamente en que la cohorte se trata como un efecto aleatorio. El propósito de dicho modelo

11 Este patrón ciertamente cambiaría si consideráramos la razón entre votantes y personas inscritas antes y después del cambio institucional de 2012. 
es robustecer la validez del análisis EPC, ya que corrobora —en virtud de la similitud que hay entre los coeficientes de ambos modelos- que el supuesto de independencia entre los efectos aleatorios de las cohortes y la edad no es violado. Esto no debiese sorprender dado el patrón marcadamente no lineal de los efectos cohorte. ${ }^{12}$ Con esta garantía en mano consideramos el modelo 3 que extiende al modelo 2 al permitir que el coeficiente de edad varíe aleatoriamente entre las distintas cohortes. Con esta flexibilización, el modelo 3 obtiene la mejor bondad de ajuste (medido por medio del Criterio de Información de Akaike) de los tres modelos descritos.

Consistente con nuestra hipótesis 2, el efecto promedio de la edad es significativo, positivo y curvilíneo, tal como lo indican los tres modelos. Es decir, controlando por las diferencias generacionales de los encuestados, la propensión a votar aumenta en la medida que los individuos envejecen, aunque la magnitud de este efecto decrece con el paso de los años. Luego, y consistente con los resultados descriptivos de la Figura 2, el modelo 3 también indica que el efecto etario presenta un nivel considerable de variación entre cohortes. En este modelo, el coeficiente estimado (promedio para todos los individuos) del término lineal de edad es de 0,62 , mientras que una desviación estándar del efecto etario entre las distintas cohortes equivale a un $82 \%$ de dicho coeficiente ().

La Figura 4 presenta las probabilidades predichas promedio de haber participado en la última elección según la edad y cohorte de nacimiento de los individuos, mientras que todas las demás variables se fijan en su valor observado. Aquí destacan dos resultados. Primero, las predicciones del modelo 3 indican que para ninguna cohorte existe una edad en la cual la propensión a votar comience a decaer, ya que las curvas siempre permanecen positivas, al menos para los tramos etarios observados (18 a 85 años). Lo anterior no descarta nuestra hipótesis 2, únicamente refleja que la tasa de cambio del efecto de edad sobre la probabilidad de votar no es lo suficientemente pronunciada para que comience a reducir las chances de votar.

Segundo, la Figura 4 también nos permite abordar nuestra hipótesis 2A, referida al efecto diferencial de la edad para las cohortes nacidas antes y después de 1970. Como puede observarse, las trayectorias etarias varían marcadamente según cohorte. Para los nacidos hasta 1935, la participación electoral es alta y relativamente estable, reflejándose en que el rango de variación de las probabilidades predichas para estas tres cohortes no supera 0,03 puntos de probabilidad. Para las cohortes nacidas entre 1936 y 1970 se aprecia una tendencia levemente positiva y curvilínea asociada a la edad. Para estas siete cohortes, las probabilidades predichas de participación electoral son altas desde un inicio (dada la inscripción electoral masiva para el plebiscito de 1988), pero,

12 Bajo el supuesto de que el modelo 1 es consistente, ya que no adolece problemas de correlación entre los efectos aleatorios de las cohortes y la edad, es posible emplear un test de tipo Hausmann (Yang y Land 2013, 204), que evalúa si los parámetros del modelo 2 son también consistentes. Los resultados aceptan la en forma clara $(\mathrm{H}=5,98 ; \mathrm{gl}=5 ; p=0,31)$. 
de todos modos, crecen gradualmente con el paso de los años. Sin embargo, dicho cambio es modesto: la diferencia promedio entre la mayor y menor probabilidad predicha para estas cohortes es de 0,10 puntos de probabilidad.

Figura 4. Probabilidades predichas promedio de participación electoral según edad y cohortes
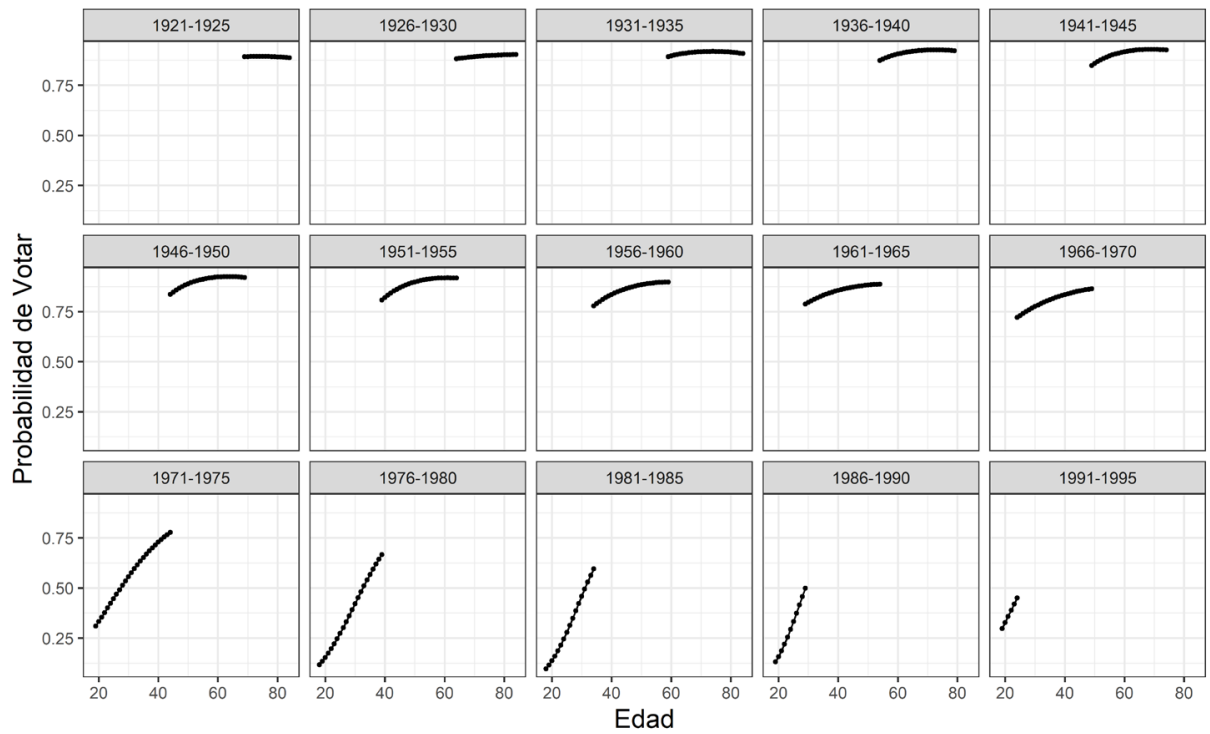

Fuente: elaboración propia en base a encuestas CEP (1994-2015)

Por último, las cohortes más jóvenes (nacidos de 1971 en adelante) registran niveles de participación electoral iniciales muy bajos, pero, con el incremento de la edad, registran un alza mucho más acentuada que el observado entre las cohortes más antiguas, en correspondencia con la hipótesis 2A. Como ejemplo, para los encuestados nacidos entre 1976 y 1980, la probabilidad promedio de votar a los 18 años era un escaso 0,12\%. Seis años más tarde -lapso tras el cual trascurrió una elección presidencial-, la probabilidad se incrementa a un $0,24 \%$, la que, si bien sigue siendo baja, implica un incremento de un $100 \%$. Al cumplir los 35 años, la probabilidad de voto de los entrevistados ha aumentado a 0,57\%, prácticamente el quíntuple de su valor original. Dicho patrón se replica en forma muy similar en todas las cohortes nacidas después de 1971. De este modo, se confirma que, para aquellas cohortes que pudieron tomar la decisión de votar en el contexto democrático postransición, hay un pronunciado efecto etario, el cual es consistente con la evidencia internacional. 


\section{DISCUSIÓN Y CONCLUSIONES}

Por medio de un análisis de edad, período y cohorte, mostramos que, entre 1994 y 2015, cada una de las fuentes de cambio social ha ejercido un efecto importante sobre la propensión a votar de los chilenos. Primero, existe un efecto cohorte marcadamente no lineal, donde la tendencia promedio a votar crece linealmente entre la cohorte más antigua (1921-1926) y la última que alcanzó a votar en el plebiscito (1966-1970). En contraste, el nivel de votación baja pronunciadamente para las generaciones que se volvieron elegibles en democracia y, particularmente, aquellas que alcanzaron la mayoría de edad entre 1990 y 1999. No obstante, la cohorte más joven (nacida entre 1991 y 1995), que coincide con quienes vivieron sus años impresionables en las movilizaciones estudiantiles de 2006 y 2011, registra un alza en los niveles de participación -aunque futuros estudios deberán confirmar si el alza se mantiene o no en el tiempo-.

Segundo, existe un claro efecto del aumento en la edad: la propensión a votar de quienes nacieron después de 1935 aumenta a medida que dichas cohortes envejecen. Dada la inscripción masiva ocurrida en 1988 y el antiguo sistema de inscripción electoral, la propensión a votar de los miembros de las cohortes previas a 1971 creció, aunque levemente. En cambio, el efecto etario adquiere una magnitud muy fuerte entre las cohortes que se volvieron elegibles para votar en democracia. Por último, constatamos la existencia de un efecto período negativo, lineal y pronunciado. Esto implica un declive progresivo y generalizado en la propensión a votar de todos los grupos etarios.

Considerados en su conjunto, estos resultados permiten derivar algunas implicancias teóricas interesantes. Primero, el patrón lineal creciente entre la cohorte más antigua (1921-1926) y la última que participó en el plebiscito de 1989 (1966-1970), confirma la existencia de un efecto propiamente generacional. Todas estas cohortes estuvieron expuestas a la obligatoriedad del voto durante el periodo bajo observación, pero las fuertes diferencias entre ellas coinciden con la cercanía a los años de socialización temprana de cada cohorte al momento del plebiscito. En contraste, no es claro si la diferencia entre las cohortes pre y postplebiscito puede atribuirse a la barrera institucional del voto obligatorio, a una estampa generacional de menor politización o a ambos. El aumento en la tasa de votación de la cohorte más reciente (1991-1995) podría favorecer el argumento generacional, pero es también cierto que esta cohorte se volvió adulta una vez que la nueva normativa de voto voluntario fue promulgada. Futuras investigaciones que contrasten detalladamente a miembros de las cohortes colindantes que alcanzaron o no a participar del plebiscito, por ejemplo, vía matching, podrían ayudar a zanjar esta incógnita.

Segundo, el efecto negativo de las cohortes postplebiscito se ha visto compensado por el hecho de que sus miembros votan más a medida que envejecen y, por ende, incrementan el promedio de votación de la cohorte con el paso del tiempo. Más aun, de no ser por el efecto etario - particularmente visible entre las cohortes 
postplebiscito, pero extensible a todas aquellas nacidas después de 1936-, el declive electoral en Chile habría sido más acentuado aún. Tercero, los resultados sugieren que el electorado chileno ha estado marcado por una suerte de patrón intracohorte divergente. Por un lado, todas las cohortes nacidas después de 1935 han aumentado su nivel de votación en razón de una dinámica etaria pero, por otro, una porción creciente de todas las cohortes, vía efecto período, ha dejado de votar. Esto refleja, posiblemente, un patrón de politización polarizado de la sociedad chilena, donde hay un segmento que se aleja progresivamente de la política y otro que, con la maduración etaria, se vuelve más cercano al mundo político.

Cuarto, aunque se insiste que los jóvenes votan menos que los mayores, nuestros resultados indican que, a medida que se vuelven adultos, votan más. En simple, los jóvenes tienden a votar menos porque son jóvenes. Este hallazgo nos obliga a reformular nuestra concepción de la juventud y la política y a preguntarnos acerca de los mecanismos que subyacen a este efecto del ciclo de la vida. La literatura internacional ofrece algunas pistas, ya sea por la vía de la maduración o el hábito, pero es perfectamente posible que el mecanismo que explica la dinámica etaria sea distinto para las diferentes cohortes.

Por último, los resultados de nuestra investigación también tienen múltiples implicancias que van más allá del caso chileno. Hasta donde conocemos, es el primer estudio EPC sobre participación electoral para un país latinoamericano, por lo que amplía la agenda sobre el votante latinoamericano (Carlin et al. 2015) y la vincula con investigaciones desarrolladas en democracias industrializadas (Grasso 2014; Smets y Neundorf 2014; Dassonneville 2017). Más aun, el patrón positivo y curvilíneo del efecto etario es consistente con los resultados de otras investigaciones sobre participación. Carlin y Love $(2015,45)$, sin un diseño explícitamente longitudinal, encuentran este mismo patrón en todos los países latinoamericanos. Esto implica que el efecto etario aquí descrito podría replicarse en otros países latinoamericanos, aunque en magnitudes potencialmente variables. En contraste, los efectos de cohorte observados no se condicen necesariamente con los detectados en otros países analizados, ya que los eventos que marcan la socialización política se vinculan fuertemente a fenómenos nacionales idiosincráticos. Sin embargo, varios países latinoamericanos experimentaron transiciones a la democracia tras dictaduras militares, por lo que podrían existir otros casos de efectos cohorte asociados a elecciones inaugurales. Más allá de las distintas formas que este efecto puede tomar, el caso chileno ejemplifica que las diferencias entre generaciones tienen un impacto duradero sobre el comportamiento político.

En la misma línea, el efecto período negativo y lineal que hemos encontrado amerita mayor atención. Aunque sea especulativo, este efecto podría deberse a la exposición acumulativa de la población a la configuración político-institucional chilena que, como han argumentado muchos académicos, no favorece el involucramiento político. El sistema electoral binomial, que erosionó el nivel de accountability del sistema político chileno (Siavelis 2009) o la consolidación 
de un sistema de partidos escasamente conectado con la sociedad civil y con bajo apoyo ciudadano (Luna y Altman 2011), podría estar detrás del declive generalizado en la propensión a votar. Una agenda de investigación comparada complementada con datos longitudinales, que se volverá crecientemente accesible con la acumulación del número de mediciones realizadas para cada país en proyectos regionales como LAPOP, hará factible abordar este tipo de hipótesis en el futuro.

\section{REFERENCIAS}

Blais, Andre. 2000. To Vote or Not to Vote? The Merits and Limits of Rational Choice Theory. Pittsburgh: University of Pittsburgh Press.

Carlin, Ryan. 2006. "The Decline of Citizen Participation in Electoral Politics in Post-Authoritarian Chile". Democratization 13 (4): 632-651.

Carlin, Ryan y Gregory Love. 2015. "Who is the Latin American Voter?". En The Latin American Voter: Pursuing Representation and Accountability in Challenging Contexts, editado por Ryan Carlin, Matthew Singer y Elizabeth Zechmeister. Ann Arbor: University of Michigan Press, 44-73.

Carlin, Ryan, Matthew Singer y Elizabeth Zechmeister. 2015. The Latin American Voter: Pursuing Representation and Accountability in Challenging Contexts. Ann Arbor: Michigan University Press.

Contreras, Gonzalo y Mauricio Morales. 2014. "Jóvenes y participación electoral en Chile, 1989-2013. Analizando el efecto del voto voluntario". Revista Latinoamericana de Ciencias Sociales, Niñez y Juventud 12 (2): 597-615.

Contreras, Gonzalo y Patricio Navia. 2013. "Diferencias generacionales en la participación electoral en Chile, 1988-2010". Revista de Ciencia Política 33 (2): 419-441.

Corvalán, Alejandro y Paulo Cox. 2013. "Class-Biased Electoral Participation: The Youth Vote in Chile". Latin American Politics and Society 55 (3): 47-68.

Czesnik, Mikolaj, Marta Zerkowska-Balas y Michal Kotnarowski. 2013. "Voting as a Habit in New Democracies: Evidence from Poland". Communist and Post-Communist Studies 46 (1): 95-107.

Dassonneville, Ruth. 2017. "Age and Voting". En The SAGE Handbook of Electoral Behaviour, editado por Kai Arzheimer, Jocelyn Evans y Michael Lewis-Beck. City Road: SAGE, 137-58.

Denny, Kevin y Orla Doyle. 2009. “Does Voting History Matter? Analyzing Persistence in Turnout". American Journal of Political Science 53 (1): 17-35.

Dinas, Elias. 2012. "The Formation of Voting Habits". Journal of Elections, Public Opinion and Parties 22 (4): 431-456.

Firebaugh, Glenn. 1997. Analyzing Repeated Surveys. Thousand Oaks: Sage.

Flanagan, Constance, Andrea Finlay, Leslie Gallay y Taehan Kim. 2012. "Political Incorporation and the Protracted Transition to Adulthood: The Need for New Institutional Inventions". Parliamentary Affairs 65 (1): 29-46.

Goerres, Achim. 2007. "Why are Older People More Likely to Vote? The Impact of Ageing on Electoral Turnout in Europe". The British Journal of Politics $\mathcal{E}$ International Relations 9 (1): 90-121.

Grasso, Maria. 2014. "Age, Period and Cohort Analysis in a Comparative Context: Political Generations and Political Participation Repertoires in Western Europe". Electoral Studies 33 (1): 63-76.

Green, Donald y Ron Shachar. 2000. "Habit Formation and Political Behaviour: Evidence of Consuetude in Voter Turnout". British Journal of Political Science 30 (4): 561-573. 
Hanmer, Michael y Kerem Ozan. 2013. “Behind the Curve: Clarifying the Best Approach to Calculating Predicted Probabilities and Marginal Effects from Limited Dependent Variable Models". American Journal of Political Science 57 (1): 263-277.

Highton, Benjamin y Raymond Wolfinger. 2001. "The First Seven Years of the Political Life Cycle". American Journal of Political Science 45 (1): 202-209.

Krosnick, Jon y Duane Alwin. 1989. "Aging and Susceptibility to Attitude Change". Journal of Personality and Social Psychology 57 (3): 416-425.

Lijphart, Arend. 1997. "Unequal Participation: Democracy's Unresolved Dilemma". The American Political Science Review 91 (1): 1-14.

Luna, Juan P. y David Altman. 2011. “Uprooted but Stable: Chilean Parties and the Concept of Party System Institutionalization". Latin American Politics and Society 53 (2): 1-28.

Madrid, Sebastián. 2005. “¿Políticos de ayer, apáticos de hoy? Generaciones, juventud y política en Chile". En El ciudadano a las urnas: un debate sobre la participación electoral en Chile, editado por Claudio Fuentes y Andrés Villar. Santiago: Facultad Latinoamericana de Ciencias Sociales, 45-83.

Manheim, Karl. 1952. "The Problem of Generations". En Essays on the Sociology of Knowledge, editado por Paul Kecskemeti. London: Routledge and Kegan Paul, 276-320.

Mason, Karen, William Mason, Haliman Winsborough y Kenneth Poole. 1973. "Some Methodological Issues in Cohort Analysis of Archival Data". American Sociological Review 38 (2): 242-58.

Milbrath, Lester. 1965. Political Participation. Chicago: Rand McNally.

Navia, Patricio. 2004. "Participación electoral en Chile, 1988-2001." Revista de Ciencia Política 24 (1): 81-103.

Parker, Cristián. 2000. Los jóvenes chilenos. Cambios culturales, perspectivas del siglo XXI. Santiago: MIDEPLAN.

Plutzer, Eric. 2002. "Becoming a Habitual Voter: Inertia, Resources, and Growth in Young Adulthood". The American Political Science Review 96 (1): 41-56.

Plutzer, Eric. 2018. "Demographics and the Social Bases of Voter Turnout". En The Routledge Handbook of Elections, Voting Behavior and Public Opinion, editado por Justin Fisher, Edward Fieldhouse, Mark Franklin, Rachel Gibson, Martha Cantijoch y Christopher Wlezien. New York: Routledge, 69-82.

Programa de las Naciones Unidas para el Desarrollo. (PNUD). 2016. Participación electoral. Chile en perspectiva comparada 1990-2016. Santiago: Área de Gobernabilidad Democrática. Recuperado de http://www.cl.undp.org/content/chile/es/home/library/democratic_governance/Participacion_electoral_02Nov2016.html

Raudenbush, Stephen y Anthony Bryk. 2002. Hierarchical Linear Models. Thousand Oaks: Sage.

Rubin, Donald. 2004. Multiple Imputation for Nonresponse in Surveys. Hoboken: John Wiley \& Sons.

Siavelis, Peter M. 2009. “Elite-Mass Congruence, Partidocracia and the Quality of Chilean Democracy". Journal of Politics in Latin America 1 (3): 3-31.

Smets, Kaat. 2016. "Revisiting the Political Life-Cycle Model: Later Maturation and Turnout Decline Among Young Adults". European Political Science Review 8 (2): 225-249.

Smets, Kaat y Carolien van Ham. 2013. "The Embarrassment of Riches? A Meta-Analysis of Individual-Level Research on Voter Turnout". Electoral Studies 32 (2): 344-359.

Smets, Kaat y Anja Neundorf. 2014. "The Hierarchies of Age-Period-Cohort Research: Political Context and the Development of Generational Turnout Patterns". Electoral Studies 33 (1): 41-51.

Strate, John, Charles Parrish, Charles Elder y Coit Ford. 1989. "Life Span Civic Development and Voting Participation". American Political Science Review 83 (2): 443-464.

Toro, Sergio. 2008. "De lo épico a lo cotidiano. Jóvenes y generaciones políticas en Chile". Revista de Ciencia Política 28 (3): 143-160.

Van Buuren, Stef. (2012). Flexible imputation of missing data. Chapman and Hall/CRC.

Verba, Sidney y Norman Nie. 1972. Participation in America: Political Democracy and Social Equality. Chicago: University of Chicago Press. 
Von Hippel, Paul. 2007. “Regression with Missing Ys: An Improved Strategy for Analyzing Multiply Imputed Data". Sociological Methodology 37 (1): 83-117.

Yang, Yang y Kenneth Land. 2013. Age-Period-Cohort Analysis: New Models, Methods, and Empirical Applications. New York: Chapman \& Hall/CRC.

Zeglovits, Eva y Julian Aichholzer. 2014. "Are People More Inclined to Vote at 16 than at 18 ? Evidence for the First-Time Voting Boost Among 16- to 25-Year-Olds in Austria". Journal of Elections, Public Opinion and Parties 24 (3): 351-361.

Recibido: 6 de octubre de 2017 Aceptado: 8 de febrero de 2018

Matías Bargsted es Ph.D. en Ciencias Políticas por la Universidad de Michigan (Ann Arbor, EE. UU.). Es profesor asistente del Instituto de Sociología en la Pontificia Universidad Católica de Chile e investigador asociado del Centro de Estudios de Conflicto y Cohesión Social (COEs). Sus áreas de investigación y docencia se centran en la opinión pública, comportamiento político, sociología de la religión y metodología cuantitativa. Ha publicado artículos en revistas como American Journal of Political Science, Comparative Sociology, Party Politics, y Latin American Politics and Society, así como varios capítulos de libros. Correo electrónico: mbargsted@uc.cl

Nicolás M. Somma es Ph.D. en Sociología por la Universidad de Notre Dame (EE. UU.). Es profesor asociado del Instituto de Sociología en la Pontificia Universidad Católica de Chile e investigador asociado del Centro de Estudios de Conflicto y Cohesión Social (COEs). Sus áreas de investigación y docencia son la sociología política, protesta y movimientos sociales, con foco en Chile y América Latina. Cuenta con más de treinta publicaciones, algunas de ellas en revistas como Party Politics, Latin American Politics and Society, Comparative Politics y The Sociological Quarterly. Correo electrónico: nsomma@uc.cl

Benjamín Muñoz Rojas es Magíster en Ciencia Política por la Pontificia Universidad Católica de Chile. Es investigador en el Centro de Estudios de Conflicto y Cohesión Social (coes). Ha publicado capítulos de libros acerca de nacionalización del sistema de partidos, competencia electoral local y partidos políticos de derecha. Correo electrónico: ajmunoz@uc.cl 